\title{
De la diversité des salles d'attente
}

\section{Eberhard Wolff}

PD Dr ès sc. soc., membre de la rédaction Histoire médicale

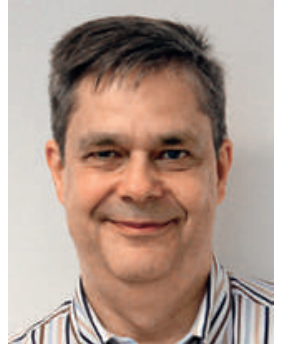

Un hôpital m’a convoqué récemment pour un examen. Peu importe ce dont il s'agissait. La vraie révélation est que j'ai traversé un dédale impressionnant de salles d'attente avant de me retrouver dehors deux heures plus tard, bien content d'être sorti.

Avant même d'avoir pu pénétrer dans le service, j'ai été retenu dans la salle d'attente $\mathrm{n}^{\circ} 1$ : une espèce de zone de préconditionnement austère, dont le rôle était de me faire remplir des formulaires avant de me laisser franchir la puissante porte de sécurité. Au sortir de cette écluse, j'ai été reçu dans la ${ }^{\circ} 2$, une «niche de pré-attente» plus confortable, équipée de fauteuils. Un médecin aimable m'y a informé de la nature et des risques de l'examen et j'ai donné mon consentement, sans doute conquis par l'atmosphère sereine et sérieuse qui se dégageait de ces lieux.

Je devais encore recevoir un premier traitement avant l'examen proprement dit, mais pas avant d'avoir subi le test de résistance qui m'attendait à la salle d'attente $n^{\circ}$ 3: une rangée de chaises dures pour pauvres pénitents alignées dans le corridor devant la salle de traitement - une impression de provisoire dans un cadre sordide, sans aucun souci de discrétion. Vous connaissez certainement ces lieux de misère qui ne méritent guère le nom d'aire d'accueil.

Quoi qu'il en soit, c'est là que j'ai su qu'il y avait un problème. L'un des appareils était bêtement tombé en panne. Or, le service de rechange n'avait rien prévu pour gérer un afflux supplémentaire de patients. Je me suis donc retrouvé dans une salle d'attente $n^{\circ} 4$ improvisée à la hâte: une salle d'examen momentanément inoccupée, où j'ai au moins pu patienter sur le lit d'examen. J'y ai appris que la notion de provisoire pouvait être étendue à l'infini.

1 L'art de prendre son mal en patience dans les salles d'attente a fait l'objet d'un article récemment paru dans la NZZ, à lire sur www.nzz.ch/ feuilleton/da-gehtnichts-weiter1.18451937

- Voir aussi: www.bmj.com/ content $/ 349 / \mathrm{bmj}$ g7262

eberhard.wolff[at]saez.ch
Fort de cette expérience, c'est dans la salle d'attente $\mathrm{n}^{\circ} 5$ (la dernière!) que j'ai retrouvé une certaine normalité: pas de chaises alignées à la va-vite, mais une vraie salle relativement accueillante qui m'a fait un peu oublier mon périple avant de passer enfin en salle d'examen.

Dans le jargon d'anthropologie culturelle, une salle d'attente est un lieu de passage, un espace de transition comme le sont les ponts et les gares. Les personnes qui s'y trouvent ont vocation à la quitter à brève échéance. Du point de vue pragmatique du personnel médical, la salle d'attente est un lieu où l'on réunit les patients pour les retrouver au moment venu sans qu'ils ne dérangent trop en attendant. Nous ne pouvons pas exiger d'une salle d'attente plus que ne l'autorisent les contraintes de l'architecture et celles encore plus pressantes des nécessités. Il reste que toute salle d'attente offre une certaine marge de manœuvre pour son agencement. Et celui-ci est parfois révélateur. J’ai eu un jour l'idée de passer en revue les salles d'attente qui m'avaient marqué personnellement.

Il y a par exemple la clinique spécialisée moderne de la ville où je vis, avec ses aires d'attente meublées de fauteuils qui font très sérieux. Je m'y sens à chaque fois comme devant la porte d'embarquement B73. Je connais un cabinet communautaire logé dans un ancien appartement inhabité d'un immeuble bourgeois. La salle d'attente, aménagée dans une pièce séparée devant la porte de l'appartement, est accessible directement depuis la cage d'escalier. On est encore «dehors» et pour être vu par le médecin, il faut d'abord être invité à entrer. On entre donc dans le «sanctuaire» sitôt que l'on passe la porte de l'appartement. L'ambiance y est radicalement différente: plus distante, plus mystérieuse et extrêmement sérieuse à la fois.

Une autre salle d'attente qui n'existe plus est restée très présente dans mon souvenir à cause de la hauteur incroyable de son plafond. La salle avait été construite à l'origine pour tester des orgues et l'on avait toujours le sentiment que rien n'était trop haut dans ce qu'on pouvait y faire.

Je me souviens enfin d'un généraliste dont la salle d'attente est le centre d'attraction architectural et l'âme de tout son cabinet. Un lieu de rendez-vous entre égaux. Tout ce qui n'a pas strictement sa place en salle de traitement y est discuté sur un ton ouvert comme nulle part ailleurs. Le médecin a beaucoup de patients d'origine ibérique et je m'y sens un peu comme sur la Plaza Mayor.

Et si vous faisiez comme moi? Comparez votre salle d'attente à celle des autres et réfléchissez au qualificatif que vous pourriez lui donner. Je pense par exemple à la salle d'attente de l'hôpital pédiatrique de Zurich, où règne ce que j'appellerais affectueusement un «joyeux tohu-bohu». Nul doute que vous verrez ensuite la vôtre avec d'autres yeux [1]. 